
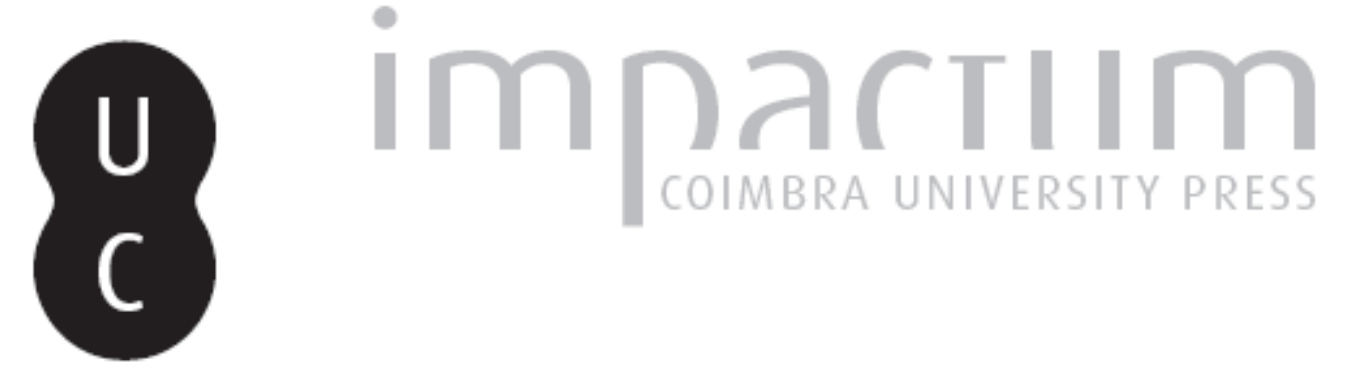

\title{
La circulación del aureo en la Hispania del siglo III d.C.
}

Autor(es): $\quad$ Sagredo San Eustaquio, Luis

Publicado por: Imprensa da Universidade de Coimbra

URL persistente:

URI:http://hdl.handle.net/10316.2/45612

DOI:

DOI:https://dx.doi.org/10.14195/1647-8657_25_6

Accessed : $\quad$ 26-Apr-2023 15:04:40

A navegação consulta e descarregamento dos títulos inseridos nas Bibliotecas Digitais UC Digitalis, UC Pombalina e UC Impactum, pressupõem a aceitação plena e sem reservas dos Termos e Condições de Uso destas Bibliotecas Digitais, disponíveis em https://digitalis.uc.pt/pt-pt/termos.

Conforme exposto nos referidos Termos e Condições de Uso, o descarregamento de títulos de acesso restrito requer uma licença válida de autorização devendo o utilizador aceder ao(s) documento(s) a partir de um endereço de IP da instituição detentora da supramencionada licença.

Ao utilizador é apenas permitido o descarregamento para uso pessoal, pelo que o emprego do(s) título(s) descarregado(s) para outro fim, designadamente comercial, carece de autorização do respetivo autor ou editor da obra.

Na medida em que todas as obras da UC Digitalis se encontram protegidas pelo Código do Direito de Autor e Direitos Conexos e demais legislação aplicável, toda a cópia, parcial ou total, deste documento, nos casos em que é legalmente admitida, deverá conter ou fazer-se acompanhar por este aviso. 
FACULDADE DE LETRAS

INSTITUTO DE ARQUEOLOGIA

CONIMBRIGA

$V O L U M E X X V$

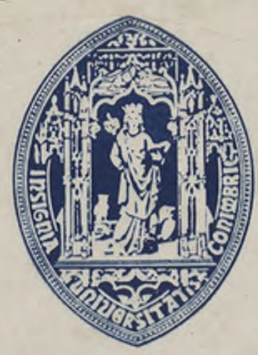

UNIVERSIDADE DE COIMBRA

1986 
Luis SAGREDO SAN EUSTAQUio

Professor da Universidade de Valladolid.

LA CIRCULACION DEL AUREO EN LA HISPANIA DEL SIGLO III D.C.

«Conimbriga», XXV (1986), p. 89-98

Resumen: La circulación del áureo en la época en estudio es francamente reducida en la Península. En su mayor parte proceden de tesorillos, es decir, que se guardaban por su valor intrínseco, y pertenecen mayoritariamente a la primera parte del siglo III d.C. Correspondiendo el mayor número de estas piezas a la etapa de los Severos y la última a Claudio II.

Summary: The Roman aurei of the third century A. D. are not common in the Iberian Peninsule. Most of the aurei come from hoards, that is, they were hidden for their own value, and they mainly belong to the earliest part of the century. Most of these coins correspond to the time of the Severi; the latest piece is an aureus of Claudius II. 
(Página deixada propositadamente em branco) 


\section{LA CIRCULACION DEL AUREO EN LA HISPANIA DEL SIGLO III D.C.}

La circulación de los áureos durante la época correspondiente al siglo ni d.C. $\left({ }^{*}\right)$ es francamente deficitaria, hay una falta casi total de ejemplares, únicamente tenemos conocimiento de 15 piezas de este tipo de monedas.

Podemos dividir los hallazgos en dos grupos pertenecientes a dos momentos cronológicos diferentes. El primero de ellos comprende los períodos correspondientes a los gobiernos que van desde el año 193 hasta mediados del siglo, concretamente hasta el final del mandato de Treboniano Galo, momento del que tenemos 10 piezas; a la segunda etapa, que abarca desde el reinado de Valeriano hasta el último momento del de Carino, corresponden otras cinco muestras.

Al primero de los grupos pertenecen una moneda de Pertinax, hallada en Bubierca $\left({ }^{2}\right)$, otra de D. Juliano hallada en Torreblanca $(3)$, siete acuñadas por Septimio Severo de las que cuatro

(x) L. Sagredo San eustaquio, Circulación e inflación monetaria en la Hispania romana del siglo III d.C., p. 1478-1489, Tesis Doctoral leída en la Universidad de Valladolid en el curso 1982-1983.

(2) J. B. LA B A Ã̃ A, Itinerario del reino de Aragón, Zaragoza, 1895, p. 129, tomado de J. P. Bost-M. Cамpo-J. M.a Gurt, "Hallazgos de aurei y solidi en la Península Ibérica: $\quad$ introducción a su circulación en época imperial», «Numisma», XXXIII, 180-185, 1983 (1986), p. 149, n. ${ }^{\circ} 30$. 138.

(3) A. D., Chronique. Trouvailles de monnaies, R. N., 1902, p. 137- 
lo son a su nombre y fueron halladas en Barroca da Lage $\left({ }^{4}\right)$, una en Torreblanco $\left(^{5}\right)$ y la otra en Lugo $\left(^{6}\right)$, dos lo fueron a nombre de I. Domna siendo encontradas una en Barroca da Lage(') y la otra en Torreblanca $\left({ }^{8}\right)$, habiendo sido acuñada la última a nombre de Geta $\left({ }^{9}\right)$. La más moderna de este primer conjunto pertenece a Filipo I y apareció en Zaragoza $\left({ }^{10}\right)$.

Es interesante comprobar que con excepción de las muestras de Pertinax y de Filipo todas las demás han sido halladas formando parte de tesorillos, uno en Portugal (u), otro en Lugo y el último en Levante. Lo que puede indicarnos que en la región oeste-noroeste, y en ese período, hubo una serie de tesorillos de colección y atesoramiento, como ocurre de forma parecida con el de Coca ( ${ }^{12}$ ), que aunque no contiene áureos que correspondan a esta etapa, sí los hay de la anterior que se continúan con los denarios, que son las modernas piezas acuñadas y con las que termina el tesorillo, dando lugar a una serie de los mismos todos ellos distintos y de buen peso $\left({ }^{13}\right)$.

En este primer grupo las piezas corresponden en su mayor parte a la época severa y una de ellas llega hasta Filipo I; las

(4) M. Heleno, O Tesouro da Borralheira (Teixoso), «O Arq. Port.», 1953 , p. 215 y 223.

(5) A. D., Chronique. Trouvaillles de monnaies..., op. cit., p. 137-138.

(6) M. Vázquez Seijas, Monumentos Arqueológicos. Numismática. Lugo y su Provincia. Libro de Oro, Lugo, 1929, p. 114-115.

(7) M. Heleno, O Tesouro da, Borralheira,..., op. cit., p. 223.

(8) A. D., Chronique. Trouvailles de monnaies..., op. cit., p. 137-138.

$\left({ }^{9}\right)$ M. Heleno, o Tesouro da Borralheira..., op. cit., p. 223.

${ }^{10}$ J. P. Bost-M. Campo-J. M. ${ }^{a}$ GuRT, Hallazgos de aurei $y$ solidi en la Península Ibérica..., op. cit., p. 173, n. ${ }^{\circ} 181$.

(u) Este hallazgo parece ser de la misma época que el del Liceo de Napoleón, ver L. Schmitt, Note sur le trésor d'aurei découvert à Paris en 1867 au lycée Napoléon, aujourd'hui Henri IV, «B.S.F.N.», 35, 10, 1980, p. 787.

(12) L. Sagredo San estaquio, Posible tesorillo del siglo ilI d.C., «Numisma», XXXI, 168-173, 1981, p. 73-88.

(13) Este tesorillo está formado por piezas de oro y plata de buena calidad; en él las monedas más antiguas son los áureos de Trajano y Adriano, que se continúan con los ejemplares de plata, denarios, hasta la época de Caracalla. 
que pertenecen a tesorillos están insertas en atesoramientos que se remontan el de Borralheira, hasta Nerón; el de Lugo, hasta Vespasiano y el de Torreblanca hasta Nerva, todo lo cual resulta normal en este tipo de hallazgos. Esto nos hace ver que las piezas anteriores a la reforma de Nerón son rarísimas (14), lo mismo ocurre con las muestras de Domiciano y Nerva, faltando igualmente, como en la mayoría de los casos, los ejemplares de Cómodo (15), que son de una extraordinaria rareza. Las muestras predominantes en la primera mitad del siglo $\mathrm{m}$ d.C. corresponden a las emisiones severas que además presentan una gran dispersión $\left.{ }^{16}\right)$.

El segundo apartado está constituido por cinco numismas que se encuentran comprendidos entre los años 258 y 270, habiendo sido emitidos 3 por Galieno, uno de los cuales pertenece a un tesorillo que comienza con Filipo I y está constituido todo él por antoninianos con la única excepción de esta pieza de oro, tesorillo aparecido en Serra do Condão (17); los otros dos están, igualmente, formando parte ya no de un tesorillo monetario, sino de un tesoro formado además por otro tipo de piezas, anillos, pendientes, lingotes de oro, etc. $\left({ }^{18}\right)$, estas muestras pertenecen a la serie de cabezas radiadas $\left({ }^{19}\right)$, que aparecen durante el reinado

(14) S. Bolín, State and Currency in the Roman Empire to 300 A.D., Stockholm, 1958, p. 191; J. P. Callu, La Politique Monétaire des Empereurs Romains de 238 à 311, Paris, 1969, p. 424-425; A. Kunisz, Ouelques remarques sur la réforme monétaire de Nerón, «Dévaluations a Rome», I, Roma, 1978, p. 89-97; D. W. MaC Dowall, The Western Coinages of Nero, New V ork, 1979, p. 31-35 y 135-140; E. Lo Cascio, La Riforma monetaria di Nerone: Vevidenza dei ripostigli, «M.E.F.R.A.», 92, 1, 1980, p. 445-470.

(15) J. P. Callu-X. Loriot, Trouvailles isolées de monnaies d'or en Gaule romaine: premières estimations, «B.S.F.N.», 35, 10, 1980, p. 783.

$\left.{ }^{16}\right)$ P. Le Gentilhomme, Les aurei du trésor découvert à Rennes en 1774. Essai sur la circulation de la monnaie d'or au IIP siécle, «R.N.», 1943, p. 20-21.

(17) M. DE Castro Hipólito, dos Tesouros de Moedas Romanas em Portugal, «Conimbriga», II-III, 1960-1961, p. 109-110.

(18) A. Ramos Folqués, Mapa arqueológico del término municipal de Elche (Alicante), «A.E.A.», XXVI, 88, 1953, p. 335 у 342.

$\left.{ }^{19}\right)$ HCC., IV, p. LIX: son los conocidos como dobles áureos, por su cabeza radiada. En 260-264, el áureo laureado se emite en menor 
de Treboniano Galo ${ }^{20}$ ), perdurando hasta la etapa de gobierno personal de Galieno, corto período en el que pierden 1,25 grs. ( $\left.{ }^{21}\right)$; las tres monedas fueron acuñadas en la segunda parte del mandato del emperador Galieno (22). De los otros dos ejemplares, uno de ellos ha sido emitido por Postumo (23) y fue hallado en Ablitas (24), mientras que el otro acuñado por Claudio II $\left({ }^{25}\right)$, apareció en Porcuna $\left({ }^{26}\right)$.

De los numismas de este segundo apartado, los de Galieno aparecen formando parte de acumulaciones monetarias, sin embargo los que pertenecen a Postumo y Claudio II corresponden al grupo de hallazgos sueltos. Solamente uno de ellos, el de Postumo, pertenece a un efnperador que gobernará fuera de Roma, concretamente en la Galia. Es interesante constatar que predominan ampliamente los ejemplares emitidos por el Imperio Central sobre los de los usurpadores, contrariamente a lo que sucede en la Galia y en Britannia, donde hay una mayor abundancia de piezas emitidas por los emperadores galos $\left({ }^{27}\right)$, como es razonable, lo cual

cantidad que el radiado acuñado en Roma para los congiaria y en Mediolanum para los soldados. Posteriormente, en 265-266, momento de la gran inflación del antoniniano en época de Galieno, el peso de las piezas laureadas cae extraordinariamente, J. P. Callu, La Politique Monétaire des Empereurs..., op. cit., p. 435 y 478.

${ }^{(20)}$ HOC., Ill, p. CIV.

(21) J. P. C. Kent, Gallienae Augustae, «N.C.», 1973, p. 65.

(22) El tipo de piezas radiadas fue introducido abundantemente, como múltiplos de oro, lo cual dio lugar a la caída del peso de los áureos laureados, L. DE BLoIs, The Policy of the Emperor Gallienus, Leiden, 1977, p. 88.

(23) Con Postumo las piezas de oro, áureos, presentan una mayor solidez, J. P. Callu, La Politique Monétaire des Empereurs..., op. cit., p. 478.

(24) F. Mateu Y Llopis, Hallazgos Monetarios III, «Ampurias», VI, 1944, p. 227, n. ${ }^{\circ}$ LXXXIII.

(25) Durante la etapa de Claudio II, se observa una revaluación del áureo, J. P. Callu, La Politique Monétaire des Empereurs..., op. cit., p. 478; HOC., IV, p. XIII.

(2e) S. de lossantos Gener, Museo Arqueológico de Córdoba, «M.M.A.P.», 1940, p. 60.

(27) F. н. тиомеsоn, A Hoard of Antoniniani from Agden near Altrincham, Cheshire, «N.C.», 1962, p. 143-155; C. G. Boon, The Erw-Hên 
nos indica que la influencia del gobierno galo sobre Hispania fue insignificante o nula. La aparición de esta última moneda en el Norte de la Península, en Ablitas, entra dentro de la lógica, ya que esta zona se encuentra más próxima al centro emisor, la Galia; otros tres ejemplares aparecieron en el Sur de Hispania, los dos radiados de Galieno y el de Claudio II, mientras que en la zona occidental tenemos un solo ejemplar, que como hemos dicho más arriba forma parte de un tesorillo monetal.

Durante el período en estudio, que cronológicamente corresponde a un siglo, casi completo, hemos visto que aparecen exclusivamente 15 piezas, las cuales se encuentran agrupadas en dos momentos del siglo, con una pieza equidistante de ambos grupos, el uno de finales del siglo $\mathrm{n}-$ comienzos del $\mathrm{m}$ y el otro de la década de los 60 .

En su mayor parte, es decir el 73,55\%, los numismas proceden de tesorillos, por lo que podemos pensar que este tipo de moneda se guardaba por su valor intrínseco y no por su valor fiduciario. Igualmente el hecho de que la mayoría de las piezas se encuentren en el primer grupo nos indica que en esa etapa hay un conjunto de ciudadanos que tienen una mayor capacidad adquisitiva, siendo sus poseedores personajes relevantes posiblemente, ya que el acceso a este tipo de moneda indicaba una categoría social desahogada, al tiempo que marcaba con un cierto prestigio (28); en el segundo apartado las muestras se encuentran más distribuidas, como muy bien pudo estarlo la posibilidad de compra tan degradada.

Contemplando ambos grupos en su entorno monetario observamos que mientras el primero se encuentra inmerso en una corriente monetal de menor entidad cuantitativa, si bien de

Treasure Trove of Roman antoniniani, «N.C.», 1966, p. 157-163; R. A. G. CARSon, Beachy Head Treasure Trove of Roman Imperial Coins at Bullock Down, «N.C.», 1968, p. 67-81; J. LAFAurie, Vempire Gaulois. Apport de la numismatique, «A.N.R.W.», II, 2, 1975, p. 853-1012; J. P. Callu-X. Loriot, Trouvailles isolées de monnaies d'or en Gaule romaine..., op. cit., p. 783; J. LA PORT, Monnaies d'or romaines dans le département $d u$ Gers, «B.S.F.N.», 37, 8, 1982, p. 283.

(28) M. Corbier, Remarques sur la circulation monétaire au III siècle, «B.S.F.N.», 35, 10, 1980, p. 796. 
mayor calidad, puesto que junto a ellos se encuentran los denarios, que aunque en estos momentos ya han sufrido los avatares de la inflación, disminución de peso y calidad ${ }^{29}$ ), todavía mantienen su fuerza en el mercado; del mismo modo que están presentes las monedas de bronce, de las que hay constancia de los tres tipos: sestercios, dupondios y ases. Los áureos de este período vemos que están incluidos dentro de una circulación plural de numismas, comprobándose a través de los tesorillos que han sido acumulados, apartados de la circulación, de acuerdo con la ley de Gresham. Por otra parte tenemos, en el segundo apartado, que las piezas áureas están rodeadas de gran cantidad de otros ejemplares, pero de mala calidad ( $\left.{ }^{30}\right)$, en su circulación.

Por consiguiente, en esta época con respecto a la anterior tenemos un aumento cuantitativo aunque no cualitativo del monetario. Es el momento en que llega la unificación de la moneda cuya base es el antoniniano; desapareciendo de la circulación todos ios demás tipos de acuñación, que se encuentran raramente y de forma casi testimonial ( ${ }^{(31)}$.

A través de lo expuesto podemos decir que durante el siglo $\mathrm{m}$ d.C. las piezas de oro se acuñaron poco y con escasez, pasándose de la época de los Severos hasta el período de Galieno, emperadores galos y Claudio II (32) con un solo ejemplar, todo lo cual entra dentro de lo racional, no siendo ajena esta laguna a la

(29) J. Guey, La dévaluation du denier romain sous Septime Sèvère, «B.S.A.F.», 1952-1953, p. 89-91; J. GuEy, La devaluation du denier sous Septime Sévere, «B.S.F.N.», 15, 1960, p. 422; J. GuEy, Ualoi du denier romain de 177 a 211 après J. C., . «R.N.», YI, 4, 1962, p. 73-140.

${ }^{(30)}$ Hay una extraordinaria calda del peso y de la ley del antoniniano durante la etapa correspondiente a los reinados de Galieno y Claudio II, cfr. P. Le Gentilhomme, Variations du titre de Vantoninianus au IIP siècle, «R.N.», VI, 4, 1962; M. ChгіsтоL, Effort de guerre et ateliers monétaires de la peripherie au IIP s. ap. J. C. Vatelier de Cologne sous $V$ alérien et Gallien, "Armées et Fiscali té dans le Monde Antique», París, 1977, p. 262; «H.C.C.», IV, p. XIII-XIV.

(31) H. HuYelin, A propos des multiples d'or de Claude II, «B.S.F.N.», 30, 2, 1975, p. 719.

(33) J. P. Callu, La Politique Monétaire des Empereurs..., op. cit., p. 428 . 
Galia (33), donde, igualmente, tanto los tesorillos de áureos como las piezas sueltas, correspondientes al período en estudio, están en inferioridad en relación con otras etapas, tanto anteriores como posteriores.

En la primera época hemos visto que predomina la forma de hallazgo en tesorillos, remontándose las piezas de estos hasta Néron $\left.{ }^{34}\right)$, siendo raras las monedas anteriores a la reforma llevada a cabo por este emperador; de Caligula no tenemos ningún ejemplar y de Nerón hay solamente un numisma emitido durante su primera etapa ( $\left.{ }^{35}\right)$, concretamente de 54-55 d.C. ( $\left.{ }^{36}\right)$. Desde el momento de su reforma hay un importante incremento de la acuñación de áureos que durará hasta el último período del siglo ii d.C., con Marco Aurelio va a tener lugar el comienzo de una prolongada y profunda caída que llevará casi a su desaparición durante la etapa que estamos analizando.

A lo largo del siglo en estudio podemos comprobar que hay una gran restricción de la circulación del oro, llegando casi a su eliminación de los circuitos monetários, lo cual queda constatado por la falta casi total de hallazgos, siendo su razón la menor producción de ejemplares de este material, así como su salida de la circulación al ser utilizados en la confección de joyas $\left({ }^{37}\right)$, pendientes, pulseras y demás objetos de exibición y de lujo. Parece ser que la utilización de los áureos montados sobre joyas se generalizó durante el curso del siglo $\mathrm{m}$ d.C., dando lugar a una forma de atesoramiento ( $\left.{ }^{38}\right)$ al tiempo que resultaba ser una moda;

(35) J. Hiernard, Las monnaies d'or romaines en Poitou, Angoumois et Saintonge, «B.S.F.N.», 35, 6, 1980, p. 709; J. P. Gallu-X. Loriot, Trouvailles isolées de monnaies d'or en Gaule romaine..op. cit., p. 783; B. Remy, Monnaies d'or romaines $d u$ departement de la Haute-Loire, «B.S.F.N.», 37, 9, 1982, p. 239.

(34) M. Heleno, O Tesouro da Borralheira.op. cit., p. 223.

(35) F. Mateu y Llopis, Hallazgos Monetarios VI, «Ampurias», XIII, 1951, p. 227, n. $^{\circ} 352$.

(36) «B.M.C.», I, p. 200-201, n. ${ }^{\circ} 4-5$.

(37) J. P. Callu, La Politique Monétaire des Empereurs..., op. cit., p. 428.

(3S) J. HiERnard, Uinterpretation des trouvailles d'aurei romains au III siécle: Vexemple des empereurs gaulois, «Studien zu Fundmünzen der Antique», 1, 1976, p. 39-77. 
esto provocó una tendencia a la desmonetización como consecuencia de la ralentización de la circulación del numerario de oro $\left.{ }^{(39}\right)$.

Los tesorillos de áureos proceden de grandes fortunas privadas, o bien de las cajas de la administración civil o militar, por lo que son las piezas aisladas las que nos indican con mayor claridad y seguridad el tipo de circulación $\left({ }^{40}\right)$.

En esta época hay una gran descentralización de las acuñaciones en función de que las emisiones siguen los movimientos del emperador, Galieno, en sus desplazamientos con su cuartel general, como consecuencia de las guerras $\left({ }^{41}\right)$.

A medida que nos adentramos en el siglo $\mathrm{m}$ el oro es sustituido en la tesaurizaciones por los denarios ( ${ }^{42}$ ) y posteriormente éstos por los nuevos tipos de acuñación, los antoninianos.

En el último tercio del siglo, que estamos analizando, hay una renovación con Aureliano, la cual tendrá un fuerte impulso con Diocleciano para desarrollarse ampliamente con Constantino.

(30) X. Loriot, Bijoux monétaires du III ${ }^{e}$ siècle. I Le collier $d u$ Vieil-Evreux (Eure), «B.S.F.N.», 38, 1, 1983, p. 265.

(4Ü) J. P. Callu-X. Loriot, Trouvailles isolées de monnaies d'or en Gaule romaine..., op. cit., p. 781 .

(41) P. Le Gentilhomme, Les aurei du trésor découvert à Rennes..., op. cit., p. 24.

(42) M. сіiristou, Effort de guerre et ateliers monétaires de la Peripherie..., op. cit., p. 253. 